\title{
Stability for a class of homogeneous hybrid systems by annular Lyapunov analysis
}

\author{
Fulvio Forni, Andrew R. Teel
}

\begin{abstract}
For a class of homogeneous hybrid systems we present a set of annular Lyapunov-like conditions for inferring global pre-asymptotic stability of systems. Then, we prove that such conditions are mild, namely, that each globally pre-asymptotically stable system must satisfy them. Based on these results, we design a sum of squares algorithm that constructs a suitable Lyapunov-like function to automatically fulfill such annular conditions. Finally, based on recent results on homogeneous approximations of hybrid systems, we point out that such conditions can also be used to deduce local preasymptotic stability of a wider class of hybrid systems.
\end{abstract}

\section{INTRODUCTION}

Hybrid systems characterize a suitable mathematical framework to model the interaction of continuous-time processes, namely processes whose dynamics depends on differential equations, with discrete-time processes, namely processes whose behavior depends on a specific transition relation. Computing devices that control mobile robots, electrical circuits that combine analog and digital components, mechanical systems with impacts, are all examples of systems that combine continuous and discrete processes and that can be conveniently characterized within the hybrid systems framework.

Several models of hybrid systems can be found in the literature, [6], [8], [13], [18]. Here we consider the framework outlined in [9] for which several structural results has been developed [11], [25], [26] and partially summarized in [10]. Although several new phenomena arise from the interaction of continuous and discrete dynamics, important results on stability theory like Lyapunov-like tools, invariance principles and converse theorems, have been generalized to the hybrid systems framework, [3], [4], [5], [7], [11], [25]. Here we propose a local Lyapunov-like approach to the study of stability properties of a particular class of homogeneous hybrid systems [30]. For instance, we define a set of Lyapunov-like conditions whose satisfaction, in a suitable subset of the state space (an annulus), guarantees global pre-asymptotic stability of the point $x_{e}=0$. We show also that such conditions are mild, that is, they are verified by any given hybrid system within the class considered, whose point $x_{e}=0$ is pre-asymptotically stable. Based on these

Research supported in part by ENEA-Euratom and MIUR. forni@disp.uniroma2.it.

Research supported in part by NSF grants CNS-0720842 and ECCS0925637 and AFOSR grant FA9550-09-1-0203. teel@ece.ucsb.edu.

F. Forni is with the Dipartimento di Informatica, Sistemi e Produzione, University of Rome Tor Vergata, 00133, Italy, forni@disp.uniroma2.it, A.R. Teel is with the Department of Electrical and Computer Engineering, University of California Santa Barbara, CA 93106-9560, USA, teel@ece.ucsb.edu. results, we define a sum of squares algorithm [21], that constructs a suitable function to automatically fulfill such conditions.

The use of sum of squares algorithms in control and, in particular, the use of sum of squares algorithms to construct Lyapunov functions, is well developed. See for example [19], [23], [28], [29]. Sum of squares formulations have been used in [1], [14], [20], [22] on arbitrary switching systems, on switched systems and on hybrid automata. In such works, the system dynamics is usually defined by polynomial functions $\dot{x}=f_{i}(x)$, or by affine functions $\dot{x}=A_{i} x+a_{i}$, for $i \in\{1, \ldots, N\}$, each of which enabled either in a subset of the state-space [14], [20], [22] or by a particular updating rule based on the state [1]. The stability analysis in [1], [14], [22] is developed by considering a part of the state that never jumps. Systems in [20] allow for the reset of the state, provided that no solutions are Zeno (namely solutions with infinitely many jumps in a bounded interval of time) or discrete (namely solutions that only jump). Then, continuous and piecewise continuous Lyapunov functions are proposed to infer stability.

The functions constructed by our algorithm satisfy usual Lyapunov conditions for pre-asymptotic stability [10] but only in an annulus. Then, for the class of systems considered, it is possible to generalize such local Lyapunov functions to the whole space, guaranteeing global pre-asymptotic stability. Our approach can be used on systems with Zeno and purely discrete solutions and produces smooth functions that may exhibit non-convex level sets in the subset of the state space in which they resembles classical Lyapunov functions. It follows that our method can be applied on global pre-asymptotically stable systems for which a convex Lyapunov function does not exists, see [2]. Finally, based on recent results on homogeneous approximation of hybrid systems [12], our method can be used on a properly defined linearization of general hybrid systems to infer local preasymptotic stability of the point $x_{e}=0$.

The work is organized as follows: in Section II, the hybrid systems framework is briefly introduced and the class of hybrid systems considered is defined. Stability concepts, main theoretical results, the sum of squares algorithm and an example are presented in Section III. Further analysis on sum of squares implementation is developed in Section IV. The conclusion follows. Proofs are in appendices.

Notation: The Euclidean norm of a vector and is denoted by $|\cdot|$. A continuous function $\alpha(\cdot):[0, a) \rightarrow[0,+\infty)$ is said to belong to class $\mathcal{K}$ if it is strictly increasing and $\alpha(0)=0$; it is said to belong to class $\mathcal{K}_{\infty}$ if $a=+\infty$ and $\lim _{r \rightarrow+\infty} \alpha(r)=+\infty$. For any given 
set $X \subset \mathbb{R}^{n}, \overline{\mathrm{co}} X$ denotes the closed convex hull of points of $X$.

\section{HYBRID SYSTEM MODEL}

We consider a model of hybrid systems given by the tuple $(C, F, D, G)$, where $C \subseteq \mathbb{R}^{n}$ and $D \subseteq \mathbb{R}^{n}$ are, respectively, the flow set and the jump set, while $F: C \rightrightarrows \mathbb{R}^{n}$ and $G$ : $D \rightrightarrows \mathbb{R}^{n}$ are set-valued mappings, respectively, the flow map and the jump map. $F$ and $G$ characterize the continuous and the discrete evolution of the system, that is, the motion of the state, while $C$ and $D$ characterize subsets of $\mathbb{R}^{n}$ where such evolution may occur. A hybrid system $\mathcal{H}$ can be conveniently represented as follows

$$
\mathcal{H}=\left\{\begin{aligned}
\dot{x} \in F(x) & x \in C \\
x^{+} \in G(x) & x \in D .
\end{aligned}\right.
$$

Intuitively, the evolution of the state either continuously flows through $C$, by following the dynamic given by $F$, or it jumps from $D$, according to $G$. Such alternation of jumps and flow intervals can be conveniently characterized by using a generalized notion of time, called hybrid time. In what follows, we recall the notions of hybrid time and of solution to a hybrid system. For details, see [9], [10], [11].

Definition 1: A set $E \subseteq \mathbb{R}_{\geq 0} \times \mathbb{N}$ is a hybrid time domain if it is the union of infinitely many intervals of the form $\left[t_{j}, t_{j+1}\right] \times\{j\}$ where $0=t_{0} \leq t_{1} \leq t_{2} \leq, \ldots$, or of finitely many such intervals, with the last one possibly of the form $\left[t_{j}, t_{j+1}\right] \times\{j\},\left[t_{j}, t_{j+1}\right) \times\{j\}$, or $\left[t_{j}, \infty\right) \times\{j\}$.

Definition 2: A hybrid arc $x$ is a map $x: \operatorname{dom} x \rightarrow \mathbb{R}^{n}$ such that (i) dom $x$ is a hybrid time domain, and (ii) for each $j$, the function $t \mapsto x(t, j)$ is a locally absolutely continuous function on the interval $I_{j}=\{t:(t, j) \in \operatorname{dom} x\}$.

A hybrid arc $x: \operatorname{dom} x \rightarrow \mathbb{R}^{n}$ is a solution to the hybrid system $\mathcal{H}$ if $x(0,0) \in C \cup D$ and

(i) for each $j \in \mathbb{N}$ such that $I_{j}$ has a nonempty interior,

$$
\begin{array}{ll}
\dot{x}(t, j) \in F(x(t, j)) & \text { for almost all } t \in I_{j} \\
x(t, j) \in C & \text { for all } t \in\left[\min I_{j}, \sup I_{j}\right)
\end{array}
$$

(ii) for each $(t, j) \in \operatorname{dom} x \operatorname{such}$ that $(t, j+1) \in \operatorname{dom} x$,

$$
\begin{aligned}
x(t, j+1) & \in G(x(t, j)) \\
x(t, j) & \in D .
\end{aligned}
$$

In what follows we consider a particular class of hybrid systems in which flow set and jump set are defined as the union of closed polyhedral cones, and flow map and jump map are defined, respectively, as the convex hull and the union of several linear vector fields. Indeed, let $i$ be an index number in $\mathbb{N}$, and let $R^{(i)}$ be a closed set defined as follows

$$
R^{(i)}=\left\{x \mid\left[\begin{array}{c}
m_{1}^{(i)} \\
\ldots \\
m_{r^{(i)}}^{(i)}
\end{array}\right] x \geq 0\right\}
$$

where $r^{(i)}$ belongs to $\mathbb{N}$ and $m_{j}^{(i)} \in \mathbb{R}^{1 \times n}$ is a row vector, for each $j=1, \ldots, r^{(i)}$. Then, $C$ and $D$ can be defined as

$$
C=\bigcup_{i \in I_{C}} R^{(i)} \quad D=\bigcup_{i \in I_{D}} R^{(i)}
$$

where $I_{C}, I_{D}$ are disjoint and finite index sets. Note that $C$ and $D$ can overlap. Note also that it is possible to have $C \cup D \neq \mathbb{R}^{n}$.

In a similar way, consider set-valued mappings $F_{i}: \mathbb{R}^{n} \rightrightarrows$ $\mathbb{R}^{n}$, for $i \in I_{C}$, and $G_{i}: \mathbb{R}^{n} \rightrightarrows \mathbb{R}^{n}$, for $i \in I_{D}$, defined as as follows. For each $i \in I_{C}, F_{i}(x)$ is a convex and closed set defined by

$F_{i}(x)=\left\{\begin{array}{cc}\overline{\operatorname{co}}\left\{f \mid f=F_{i k} x \text { for } k=1 \ldots r_{F}\right\} & \text { if } x \in R^{(i)} \\ \emptyset & \text { otherwise }\end{array}\right.$

where $F_{i k} \in \mathbb{R}^{n \times n}$ and $r_{F} \in \mathbb{N}$. For each $i \in I_{D}, G_{i}(x)$ is a set defined by

$G_{i}(x)=\left\{\begin{array}{cc}\left\{g \mid g=G_{i k} x \text { for } k=1 \ldots r_{G}\right\} & \text { if } x \in R^{(i)} \\ \emptyset & \text { otherwise }\end{array}\right.$

where $G_{i k} \in \mathbb{R}^{n \times n}$ and $r_{G} \in \mathbb{N}$. Then, flow and jump mappings, $F: \mathbb{R}^{n} \rightrightarrows \mathbb{R}^{n}$ and $G: \mathbb{R}^{n} \rightrightarrows \mathbb{R}^{n}$, can be defined as

$$
F(x)=\overline{\mathrm{co}} \bigcup_{i \in I_{C}} F_{i}(x) \quad G(x)=\bigcup_{i \in I_{D}} G_{i}(x)
$$

Note that $F(x)$ reduces to $F_{i}(x)$ when $x$ belongs only to one cone $R^{(i)}$, for some $i \in I_{C}$. The same holds for $G(x)$.

Hybrid systems of the form (1),(4)-(8) satisfy the following basic conditions. Such conditions coincide with the basic assumptions of [10] and with the fundamental conditions of [11]

Claim 1 (Basic Conditions): A hybrid system $\mathcal{H}$ of Equations (1),(4)-(8) satisfies the following properties:

1) $C \subseteq \mathbb{R}^{n}$ and $D \subseteq \mathbb{R}^{n}$ are closed sets in $\mathbb{R}^{n}$.

2) $F: \mathbb{R}^{n} \rightrightarrows \mathbb{R}^{n}$ is an outer semicontinuous set-valued mapping, locally bounded on $C$ and, for each $x \in C$, $F(x)$ is nonempty and convex.

3) $G: \mathbb{R}^{n} \rightrightarrows \mathbb{R}^{n}$ is an outer semicontinuous set-valued mapping, locally bounded on $D$ and, for each $x \in D$, $G(x)$ is nonempty.

Proof: $C$ and $D$ are finite union of closed sets. Boundedness of $F$ and $G$ follows from the fact that they are constructed from linear vector fields. Convexity of $F(x)$, for each $x \in C$, follows from the use of the convex-hull operator. Finally, outer semicontinuity of $F$ follows from the fact that its graph is closed. Thus, by [24, Theorem 5.7] $F$ is outer semicontinuous. Analogously for $G$.

Remark 1: Hybrid systems that satisfy the conditions in Claim 1 exhibit a sort of regularity of solutions that leads to several important results. For example, for such systems, sequential compactness of the space of solutions holds, [11, Theorem 4.4 and Lemma 4.3], there is outer semicontinuous dependence of solutions on initial conditions, [10, Theorem 5] and [11, Corollary 4.8], and it is possible to relate solutions to a hybrid system $\mathcal{H}$, with solutions to stateperturbed hybrid systems $\mathcal{H}_{\delta}$, constructed from $\mathcal{H}$ by a suitable state-perturbation of magnitude $\delta$, [10, Theorem 8] and [11, Theorem 5.1]. Regularity of solutions has effects also on stability theory. See [11], [25] 
Remark 2: Switched linear systems with state dependent switching policies, [15, Sections 3.3 and 3.4], can be characterized within the family of hybrid systems considered above. For example, consider the system

$$
\dot{x}=A_{i} x \text { if } x \in C_{i}, \quad i=1, \ldots, N .
$$

where $N \in \mathbb{N}$ and, for each $i=1, \ldots, N, A_{i} \in \mathbb{R}^{n \times n}$ and $C_{i}$ is a conic subset of $\mathbb{R}^{n}$. Such systems can be easily defined within the class of hybrid systems considered above, by defining $F_{i}(x)=A_{i} x$ if $x \in C_{i}$ and $F_{i}(x)=\emptyset$ otherwise, for each $i=1, \ldots, N$. In such case, $D=\emptyset$. Moreover, switched linear systems under arbitrary switching policies, [15, Section 2.1.4], can be written as hybrid systems (1),(4)(8), based on a single differential inclusion of the form (6), defined by the convex hull of the linear vector fields of the switched linear system, and $C=\mathbb{R}^{n}$.

\section{Stability}

\section{A. Preliminaries}

By following [10], for a hybrid system $\mathcal{H}$, the point $x_{e}=0$ is (i) stable if for each $\epsilon>0$ there exists $\delta>0$ such that any solution $x$ to $\mathcal{H}$ with $|x(0,0)| \leq \delta$ satisfies $|x(t, j)| \leq \epsilon$ for all $(t, j) \in \operatorname{dom} x$; (ii) pre-attractive if there exists $\delta>0$ such that any solution $x$ to $\mathcal{H}$ with $|x(0,0)| \leq \delta$ is bounded and $x(t, j) \rightarrow 0$ as $t+j \rightarrow 0$ whenever $x$ is complete; (iii) pre-asymptotically stable if it is both pre-stable and pre-attractive. The basin of pre-attraction $\mathcal{B}_{x_{e}}$ is the set of points in $\mathbb{R}^{n}$ from which each solution is bounded and the complete solutions converge to $\mathcal{A}$. Finally, if the basin of preattraction $\mathcal{B}_{x_{e}}=\mathbb{R}^{n}$ then $x_{e}$ is globally pre-asymptotically stable. In such case we say that the system is globally preasymptotically stable.

Stability properties of a hybrid system $\mathcal{H}$ can be studied with Lyapunov-like tools. By following [10], a function $V: \operatorname{dom} V \rightarrow \mathbb{R}$ is a Lyapunov-function candidate for $\left(\mathcal{H}, x_{e}\right)$ if (i) $V$ is continuous and nonnegative in $(C \cup D) \backslash$ $\left\{x_{e}\right\} \subseteq \operatorname{dom} V$ and (ii) $V$ is continuously differentiable on an open set $\mathcal{O}$ satisfying $C \backslash\left\{x_{e}\right\} \subseteq \mathcal{O} \subseteq \operatorname{dom} V$, and (iii) $\lim _{x \rightarrow x_{e}, x \in \operatorname{dom} V \cap(C \cup D)} V(x)=0$.

Theorem 1 ([10, Theorem 20]): Consider the hybrid system $\mathcal{H}=(C, F, D, G)$ satisfying Claim 1 and an equilibrium point $x_{e}$. If there exists a Lyapunov-function candidate $V$ for $\left(\mathcal{H}, x_{e}\right)$ such that

$$
\begin{aligned}
\langle\nabla V(x), f\rangle<0 & \text { for all } x \in C \backslash\left\{x_{e}\right\}, f \in F(x), \\
V(g)-V(x)<0 & \text { for all } x \in D \backslash\left\{x_{e}\right\}, g \in G(x) \backslash\left\{x_{e}\right\},
\end{aligned}
$$

then $x_{e}$ is pre-asymptotically stable and the basin of preattraction contains every forward invariant compact set. Moreover, suppose that either $C \cup D$ is a compact set, or the sublevel sets of $\left.V\right|_{C \cup D}$, defined by $\{x \in C \cup D \mid V(x) \leq c\}$ with $c \in \mathbb{R}_{\geq 0}$, are compact. Then, $x_{e}$ is globally preasymptotically stable

In what follows we show some results on stability of equilibrium point $x_{e}$ of hybrid systems (1),(4)-(8) by following a Lyapunov-like approach, namely, by using a suitable selected function $V$ that satisfies a given set of conditions. Then, we present a sum of squares algorithm to effectively construct such function $V$.

\section{B. Main results}

Global pre-asymptotic stability of a hybrid system (1),(4)(8) can be inferred from a local analysis of the system by finding a function $V: \mathbb{R}^{n} \rightarrow \mathbb{R}$ that respects some specific conditions on the annulus $\{x|c \leq| x \mid \leq \rho c\}$, where $c \in \mathbb{R}_{\geq 0}$ and $\rho \in \mathbb{R}_{>1}$. The local satisfaction of such conditions will guarantee global pre-asymptotic stability of the system, as stated in the following theorem.

Definition 3: A function $\vartheta: \mathbb{R}^{n} \rightarrow \mathbb{R}$ is said to be homogeneous of degree $k \in \mathbb{N}$ if for all $x \in \mathbb{R}^{n}$ and all $\lambda \in \mathbb{R}_{\geq 0}, \vartheta(\lambda x)=\lambda^{k} \vartheta(x)$,

Theorem 2: For a hybrid system $\mathcal{H}$ (1),(4)-(8), suppose that there exist a function $V: \mathbb{R}^{n} \rightarrow \mathbb{R}$ and constants $c \in$ $\mathbb{R}_{>0}$ and $\rho \in \mathbb{R}_{>1}$ such that,

- for each $x$ in $\{x|c \leq| x \mid \leq \rho c\}$,

$$
\begin{array}{ll}
\text { (1) }\langle\nabla V(x), f\rangle<0 & x \in C, \forall f \in F(x) \text {; } \\
\text { (2) } V(g)-V(x)<0 & x \in D, \forall g \in G(x) \text {; }
\end{array}
$$

- there exists $\ell_{1}, \ell_{2} \in \mathbb{R}_{>0}, \ell_{1}<\ell_{2}$,

(3) $\max _{|x|=c} V(x) \leq \ell_{1}$ and $\min _{|x|=\rho c} V(x) \geq \ell_{2}$;

(4) if $x \in D \cap\{x|c \leq| x \mid \leq \rho c\} \cap\left\{x \mid V(x) \leq \ell_{2}\right\}$ and $g \in G(x)$ then $|g| \leq \rho c$;

- $V$ is a continuous function in $C \cup D$, and it is continuously differentiable in an open set that contains $C$.

Then, for any given constant $k \in \mathbb{R}_{>0}$, there exists a function $\bar{V}: \mathbb{R}^{n} \rightarrow \mathbb{R}_{\geq 0}$ and some constants $a_{2} \geq a_{1}>0, \mu>0$, $0<\nu<1$ in $\mathbb{R}$ such that $\bar{V}$ is a homogeneous function of degree $\mathcal{K}$, continuous in $\mathbb{R}^{n}$, smooth in $\mathbb{R}^{n} \backslash\{0\}$, and

$$
\begin{array}{cl}
a_{1}|x|^{k} \leq \bar{V}(x) \leq a_{2}|x|^{k} & \forall x \in C \cup D \\
\langle\nabla \bar{V}(x), f\rangle \leq-\mu \bar{V}(x) & \forall x \in C, \forall f \in F(x) \\
\bar{V}(g) \leq \nu \bar{V}(x) & \forall x \in D, \forall g \in G(x)
\end{array}
$$

Proof: See Appendix A.

The meaning of Conditions (1)-(4) of the theorem can be explained by considering Figure 1, in which we summarized the case of a planar hybrid system for which conditions (1)-(4) are satisfied. In general, Conditions (1)-(2) can be interpreted as usual Lyapunov conditions for pre-asymptotic stability but, in Theorem 2, each inequality must be satisfied only for $c \leq|x| \leq \rho c$. For guaranteeing a global result from such annular Lyapunov-like conditions (1)-(2), we need some extra-conditions on the function $V(x)$ and on the dynamics of the system. With this aim, $\ell_{1}<\ell_{2}$ and Condition (3) force $V(x)$ to be lower at $|x|=c$ than at $|x|=\rho c$, guaranteeing the existence of two level sets of $V(x)$ that surround the origin. This is represented in Figure 1 by closed curves with labels $\ell_{1}$ and $\ell_{2}$. By looking at the figure, Condition (4) ensures that no jumps from a state within the set enclosed by $\ell_{2}$ can bring the state out of $\rho c$.

Conditions in Theorem 2 are quite mild, as stated in the following theorem, whose proof is in Appendix B.

Theorem 3: For a a hybrid system $\mathcal{H}$ (1),(4)-(8), the equilibrium $x_{e}=0$ is globally pre-asymptotically stable if and only if there exists a smooth function $V: \mathbb{R}^{n} \rightarrow \mathbb{R}$ that 


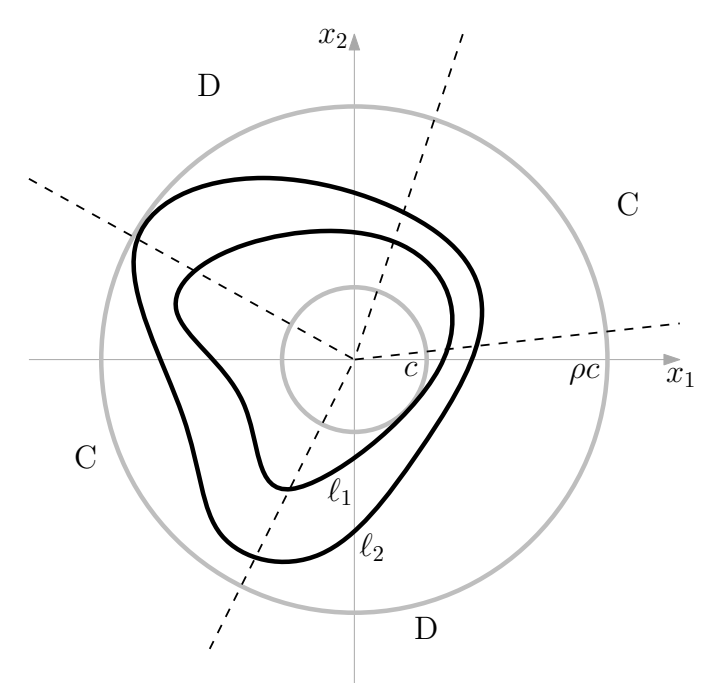

Fig. 1. A function $V$ that satisfies Theorem 2 for a planar hybrid system.

satisfies Conditions (1)-(4) of Theorem 2, for some $c \in \mathbb{R}_{>0}$ and $\rho \in \mathbb{R}_{>1}$.

Remark 3: Following [12], for general a hybrid system $\mathcal{H}$, (1), that satisfies the basic conditions, local pre-asymptotic stability of the point $x_{e}=0$ can be deduced from the preasymptotic stability of $x_{e}=0$ of a suitable approximation $\mathcal{H}_{L}$ of $\mathcal{H}$. Therefore, Theorem 2 can be used to infer local pre-asymptotic stability of $x_{e}=0$ of a general hybrid system $\mathcal{H}$ whose approximation $\mathcal{H}_{L}$ is definable within the class of hybrid systems of equations (1),(4)-(8) and satisfies the conditions of Theorem 2 . Indeed, following [12, Theorem 3.16], Theorem 2 can be applied to homogeneous approximations $\mathcal{H}_{L}$ of hybrid systems $\mathcal{H}$ based on a dilation $M(\lambda)=\lambda I$ ([12, Definition 3.7]), such that (i) tangent cones $T_{C}\left(x_{e}\right)$ and $T_{D}\left(x_{e}\right)$ are polyhedral cones ([12, Definition 3.9]) and (ii) set-valued mappings $\overline{\mathrm{co}} F^{M, 0}$ and $G^{M, 0}$ are definable as combinations of a finite number of linear vectors field ([12, Definition 3.13]).

\section{Sum of squares algorithm}

In this section we present an algorithm for finding a function $V$ that satisfies the conditions of Theorem 2. The general idea is to construct a set of polynomial inequalities that imply the conditions of the theorem. Then, a solution to such set of inequalities is computed by (i) relaxing each inequality to a sum of squares decomposition, and by (ii) using a semidefinite program solver for seeking a solution to the whole sum of squares decomposition problem. Of course, conservativeness is introduced [21], [23]. Algorithm 1 works as follows. The input of the algorithm is filled by the data of the hybrid system $\mathcal{H}$ and by parameters $\varepsilon, c, \rho, d_{1}$ and $d_{2}$, as stated in INPUT. A set of inequalities is then constructed, parameterized on $\mathcal{H}$ and on $\varepsilon, c, \rho, d_{1}$ and $d_{2}$, as stated in CONSTRAINTS. Each inequality uses variables defined in VARIABLES. Then, a solution is computed by relaxing the satisfiability problem of the whole set of inequalities to a sum of squares decomposition problem. A semidefinite program solver runs over such problem and, if it finds a solution, the set of inequalities is feasible and the algorithm ends positively, as stated in OUTPUT.

The following quantities are used in the algorithm.

Definition 4: For any given $i \in I_{C} \cup I_{D}$, the function $\Delta_{2}^{(i)}(x): \mathbb{R}^{n} \rightarrow \mathbb{R}$ is defined as follows

$$
\begin{aligned}
\Delta_{2}^{(i)}(x) & =\sum_{j=1}^{r^{(i)}} p_{j}(x) m_{j}^{(i)} x+\sum_{j=1}^{r^{(i)}} \sum_{k=j+1}^{r^{(i)}} p_{j k}(x) m_{j}^{(i)} x m_{k}^{(i)} x \\
& +\sum_{j=1}^{r^{(i)}} \sum_{k=j+1}^{r^{(i)}} \sum_{h=k+1}^{r^{(i)}} p_{j k h}(x) m_{j}^{(i)} x m_{k}^{(i)} x m_{h}^{(i)} x \\
& +\ldots+p_{1,2, \ldots, r}(x) m_{1} x m_{2} x \cdot \ldots \cdot m_{r^{(i)}} x
\end{aligned}
$$

where, for any given combination of indices $j, k, \ldots$, $p_{j}, p_{j k}, \ldots$ denote functions in $\mathbb{R}^{n} \rightarrow \mathbb{R}_{\geq 0}$, defined by nonnegative polynomials of a given degree. We refer to the whole set of polynomials $p_{j}, p_{j k}, \ldots$ by using the name slack polynomials.

Definition 5: Let $\varepsilon_{1}, \varepsilon_{2} \in \mathbb{R}_{\geq 0}$ be two constants and let $\Delta_{1}\left(\varepsilon_{1}, \varepsilon_{2}, \cdot\right): \mathbb{R}^{n} \rightarrow \mathbb{R}$ be a map defined with respect to $\varepsilon_{1}$ and on $\varepsilon_{2}$ as

$$
\Delta_{1}\left(\varepsilon_{1}, \varepsilon_{2}, x\right)=-\left(|x|^{2}-\varepsilon_{1}^{2}\right)\left(|x|^{2}-\varepsilon_{2}^{2}\right) .
$$

It follows from (4) that, for each $i \in I_{C} \cup I_{D}, \Delta_{2}^{(i)}(x)$ is positive for each $x$ in $R^{(i)}$ while it is possibly negative for $x \notin R^{(i)}$, based on the particular configuration of slack polynomial. $\Delta_{1}\left(\varepsilon_{1}, \varepsilon_{2}, x\right)$ is positive for $\varepsilon_{1} \leq|x| \leq \varepsilon_{2}$, and is strictly negative otherwise. A planar example of a subset of $\mathbb{R}^{n}$ with positive $\Delta_{1}$ and $\Delta_{2}$ is in Figure 2. $\Delta_{1}$ and $\Delta_{2}$ are used in Algorithm 1 for relaxing the conditions on $V$ to hold only in a subset of $\mathbb{R}^{n}$.

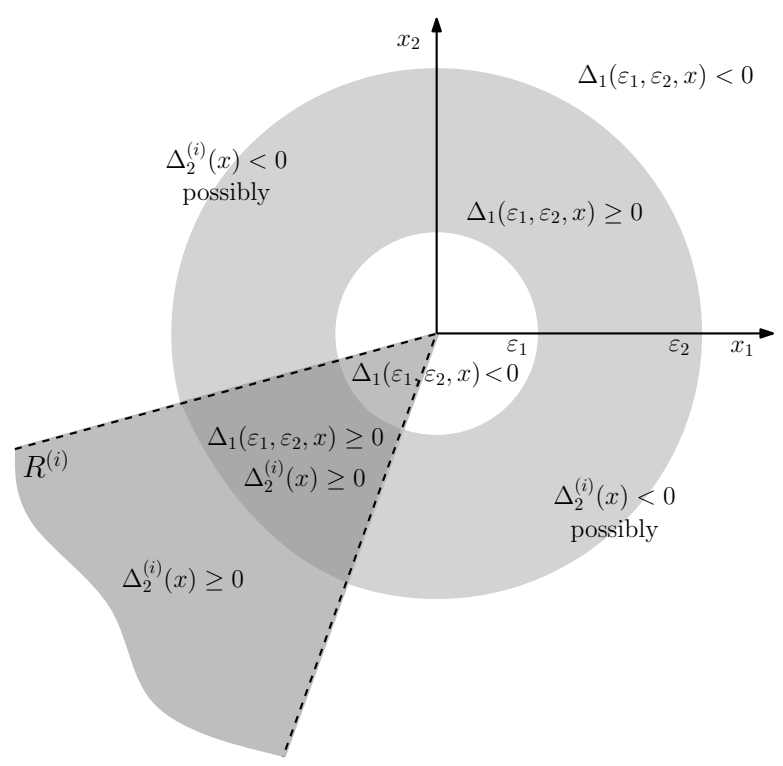

Fig. 2. Subsets of the state-space related to the sign of $\Delta_{1}$ and $\Delta_{2}$.

\section{Algorithm 1:}

INPUT : data $\langle F, G, C, D\rangle$ of the hybrid system $\mathcal{H}$; constants $\varepsilon, c, \rho \in \mathbb{R}_{>0}$, satisfying $\varepsilon \ll c$ and $\rho>1$; 
constants $d_{1}, d_{2} \in \mathbb{N}$, satisfying $d_{1} \geq d_{2}$.

OUTPUT: feasibility of the sum of squares problem.

VARIABLES : scalar variables $\epsilon, \ell_{1}, \ell_{2}$; polynomials $V(x), s_{3}(x), s_{4}(x), s_{1}^{(i k)}(x)$, for each $i \in I_{C}$ and each $k=1, \ldots, r_{F}, s_{2}^{(i k)}(x), s_{5}^{(i k)}(x), s_{6}^{(i k)}(x)$, for each $i$ in $I_{D}$ and each $k=1, \ldots, r_{G}$, and all the slack polynomials.

CONSTRAINTS : $V(x)$ is a polynomial of degree $d_{2}$.

- $\forall i \in I_{C}, \forall k \in\left\{1 \ldots, r_{F}\right\}, s_{1}^{(i k)}(x)$ is a polynomial of degree $d_{1}$ and

$$
\begin{aligned}
-\nabla V(x)^{T} F_{i k} x-\Delta_{2}^{(i)}(x)-s_{1}^{(i k)}(x) \Delta_{1}(c, \rho c, x) & >0 \\
s_{1}^{(i k)}(x) & \geq 0
\end{aligned}
$$

- $\forall i \in I_{D}, \forall k \in\left\{1 \ldots, r_{G}\right\}, s_{2}^{(i k)}(x)$ is a polynomial of degree $d_{1}$ and

$$
\begin{aligned}
V(x)-V\left(G_{i k} x\right)-\Delta_{2}^{(i)}(x)-s_{2}^{(i k)}(x) \Delta_{1}(c, \rho c, x) & >0 \\
s_{2}^{(i k)}(x) & \geq 0
\end{aligned}
$$

- $s_{3}(x)$ and $s_{4}(x)$ are polynomials of degree $d_{1}, \ell_{1}$ and $\ell_{2}$ are scalar variables and

$$
\begin{aligned}
\ell_{1}-V(x)-s_{3}(x) \Delta_{1}(c, c+\varepsilon, x) & \geq 0 \\
V(x)-\ell_{2}-s_{4}(x) \Delta_{1}(\rho c-\varepsilon, \rho c, x) & \geq 0 \\
\ell_{2}-\ell_{1} & >0 \\
s_{3}(x), s_{4}(x), \ell_{1}, \ell_{2} & \geq 0
\end{aligned}
$$

- $\forall i \in I_{D}, \forall k \in\left\{1 \ldots, r_{G}\right\}, s_{5}^{(i k)}(x)$ and $s_{6}^{(i k)}(x)$, are polynomials of degree $d_{1}, \ell_{1}, \ell_{2}$ are scalar variables and

$$
\begin{aligned}
V(x)-\ell_{2}-s_{5}^{(i k)}(x)\left(x^{\prime} G_{i k}^{T} G_{i k} x-\rho^{2} c^{2}\right)+ \\
-\Delta_{2}^{(i)}(x)-s_{6}^{(i k)}(x) \Delta_{1}(c, \rho c, x) \geq 0 \\
s_{5}^{(i k)}(x), s_{6}^{(i k)}(x) \geq 0
\end{aligned}
$$

- For each use of $\Delta_{2}^{(i)}(x)$ in (10), (11) and (13) a new fresh set of slack polynomials must be used. Moreover for each slack polynomial, say $p(x)$, a new inequality $p(x) \geq 0$ is added.

Remark 4: The last bullet of Algorithm 1 requires a new set of slack polynomials for each use of $\Delta_{2}^{(i)}(x)$. For example, slack polynomials of $\Delta_{2}^{(i)}(x)$ used in an inequality that involves $G_{i k_{1}}$ in (11) must not be confused with slack polynomials of $\Delta_{2}^{(i)}(x)$ used in an inequality that involves $G_{i k_{2}}$ in (11), with $k_{1} \neq k_{2}$.

The left-hand side of each inequality of Algorithm 1 is constructed following two goals: the first part is used to enforce some constraint on $V$ so that $V$ fulfils the conditions of Theorem 2, while the second part uses $\Delta_{1}$ and $\Delta_{2}^{(i)}$, for $i \in I_{C} \cup I_{D}$, to guarantee that $V$ satisfies some constraints only in a subset of $\mathbb{R}^{n}$, leaving $V$ practically unconstrained in the rest of the space.

Consider now to run Algorithm 1 for some given hybrid system $\mathcal{H}$ and to find a feasible solution. Inequalities
(10) and (11) guarantee that (i) the directional derivative of $V(x),\langle\nabla V(x), f\rangle$, is negative for each $x$ in the set $C \cap\{x|c \leq| x \mid \leq \rho c\}$ and each $f \in F(x)$, and (ii) the increment of $V(x), V(g)-V(x)$, is negative for each $x$ in the set $D \cap\{x|c \leq| x \mid \leq \rho c\}$ and each $g \in G(x)$. Therefore, (10) and (11) are related to Conditions (1) and (2) of Theorem 2. The first inequality of (12) implies $\max _{c \leq|x| \leq c+\varepsilon} V(x) \leq \ell_{1}$. The second inequality of (12) implies $\min _{\rho c-\varepsilon \leq|x| \leq \rho c} V(x) \geq \ell_{2}$. Note that $\ell_{1}<\ell_{2}$ by third inequality. Thus, (12) is related to Condition (3) of Theorem 2. Inequality (13) guarantees that a hybrid arc of $\mathcal{H}$ cannot escape the set $\{x|| x \mid \leq \rho c\}$ by a jump from $D \cap\{x|c \leq| x \mid \leq \rho c\} \cap\left\{x \mid V(x) \leq \ell_{2}\right\}$. Therefore, (13) is related to Conditions (4) of Theorem 2. It follows that a feasible solution to the set of constraints produces a function $V$ that satisfies the conditions of Theorem 2, as stated in the following proposition, whose proof is in Appendix C.

Proposition 1: For any given hybrid system $\mathcal{H}(1),(4)-(8)$, if the set of inequalities of Algorithm 1 has a feasible solution for some parameters $c \in \mathbb{R}_{>0}$ and $\rho \in \mathbb{R}_{>1}$, then the function $V$ constructed by Algorithm 1 satisfies each condition of Theorem 2 with the same $c$ and $\rho$.

Remark 5: Despite the number of index $i, j, k$ used during the description of Algorithm 1, in practical cases such algorithm is much more simple. For example, switched systems in [15, Sections 3.3 and 3.4] require a single matrix $F_{i}$ for each cone $R^{(i)}$. Therefore $k=1$ in (10).

Remark 6: Note that $V(x)$ is practically unconstrained when $\Delta_{1}(x)$ and $\Delta_{2}(x)$ are negative. Thus, the solver has some extra-degree of freedom during the construction of $V(x)$. Indeed, $V(x)$ that can be non-positive near the origin and non-positive far from the origin, i.e. with low order terms and high order terms not necessarily positive, that allows for the construction of functions $V(x)$ with complex and not necessarily convex level sets. In fact, it could be the case that the hybrid system (1),(4)-(8) does not have a convex Lyapunov functions, as shown in [2].

\section{Example}

We use Algorithm 1 to study the stability of a hybrid system $\mathcal{H}(1),(4)-(8)$ defined by the following quantities:

$C=C_{1} \cup C_{2}$ where $C_{1}=\left\{x \mid M_{1} x \geq 0\right\}, C_{2}=$ $\left\{x \mid M_{2} x \geq 0\right\}$, and $D=\left\{x \mid M_{3} x \geq 0\right\}$.

$$
\begin{aligned}
& F(x)= \begin{cases}F_{1} x & \text { if } x \in C_{1} \backslash C_{2} \\
\overline{\operatorname{co}}\left\{F_{1} x, F_{2} x\right\} & \text { if } x \in C_{1} \cap C_{2} \\
F_{2} x & \text { if } x \in C_{2} \backslash C_{1} \\
\emptyset & \text { otherwise }\end{cases} \\
& G(x)= \begin{cases}G x & \text { if } x \in D . \\
\emptyset & \text { otherwise }\end{cases}
\end{aligned}
$$

$F_{1}=\left[\begin{array}{cc}\frac{1}{2} & -1 \\ 1 & \frac{1}{2}\end{array}\right], F_{2}=\left[\begin{array}{cc}-\frac{1}{2} & -1 \\ 1 & -\frac{1}{2}\end{array}\right], G=\left[\begin{array}{cc}e^{\frac{1}{10}} & 0 \\ 0 & e^{-\frac{1}{10}}\end{array}\right]$,
$M_{1}=\left[\begin{array}{cc}1 & \frac{1}{200} \\ 1 & 2\end{array}\right], M_{2}=\left[\begin{array}{cc}-1 & -2\end{array}\right], M_{3}=\left[\begin{array}{cc}-1 & -\frac{1}{200} \\ 1 & 2\end{array}\right]$.
Some level set of the function $V(x)$ constructed by Algorithm 1, for $c=0.5, \rho=4.4, \varepsilon=0.001, d_{1}=10$ 
and $d_{2}=10$, are summarized in Figure 3 .

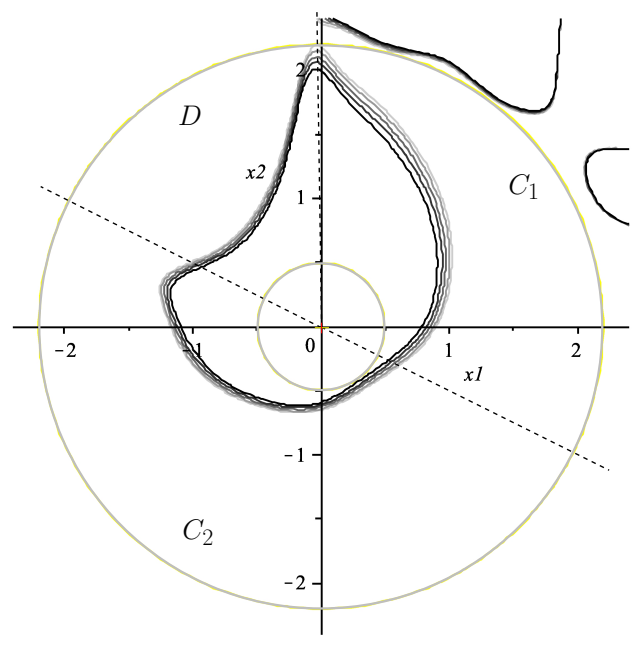

Fig. 3. Some level sets of the function $V(x)$ constructed by Algorithm 1.

The system used in this example is an adaptation to the hybrid system framework of the switching system in [2, Section 3], for which a convex Lyapunov function does not exist. In that case, by using $d_{2}=2$, the algorithm would not have enough degrees of freedom on $V$ to satisfy the set of constraints, and we need $d_{2}=10$ to construct a suitable function $V$ with non-convex level sets. Moreover, if we take $c$ too small, numerical problems occur during the construction of $V$.

\section{NOTES ON SUM OF SQUARES IMPLEMENTATION}

The problem of finding a solution to the set of inequalities of each algorithm is addressed by replacing each inequality with a sum-of-squares decomposition. In fact, the left-hand side of each inequality involving polynomials is a polynomial, say $p(x)$. It follows that inequalities $p(x) \geq 0$ can be replaced by $p(x)$ is a sum-of-squares and each strict inequality $p(x)>0$ can be considered as a non-strict inequality of the form $p(x)-\epsilon x^{T} x \geq 0$, with $\epsilon>0$ variable of the problem, then replaced by $p(x)-\epsilon x^{T} x$ is a sum-ofsquares.

From a computational point of view, finding a sum-ofsquares decomposition is much easier than using a general algorithm for finding a solution to the inequality constraints. At the same time, it could be the case that a solution to the inequality constraints exists while the sum of squares decomposition fails to exist. Moreover, even though (i) polynomial inequalities constructed by each algorithm are linear with respect to the set of variables and (ii) a sum of squares decomposition problem can be solved in polynomial time, the computational complexity of finding a solution to the set of inequalities grows rapidly with the dimension of the state-space of $\mathcal{H}$, with the degree of free polynomials used in the set of inequalities, with the number of disjoint cones of $C \cup D$, and with the number of matrices $F_{i k}, G_{i k}$.

It is worth mentioning that a sum-of-squares decomposition is satisfied within the limits of numerical computation; therefore it cannot be exact. Fortunately, we are not interested in an exact decomposition. What we really need is that, despite the numerical approximation errors, the polynomials constructed by the sum of squares decomposition are still a feasible solution to the set of inequalities. By following [17], such goal can be achieved by considering a perturbed polynomial with a perturbation magnitude that depends on the numerical approximations errors of the decomposition (residuals). Then, we can use [17, Theorem 4] to guarantee that the approximate solution to the sum of squares decomposition problem is a feasible solution for the set of inequalities. For instance, consider the case of a polynomial $p(x)$ such that $p(x) \geq 0$ : (i) we relax the problem to find $p(x)$ such that $p(x)$ is a sum-of-square; (ii) the data of the SDP formulation are matrices $A$ and $b$; (iii) the solution is $P \in \mathbb{R}^{M \times M}$, for some $M \in \mathbb{N}$; (iv) $p(x)$ can be written as $v(x)^{\prime} P v(x)$, where $v(x)$ is a base of monomials. Then, by [17, Theorem 4], if the test $\lambda_{\min }(P) \geq M\|A(P)-b\|_{\infty}$ is verified, we have that $v(x)^{\prime} P v(x)$ is non-negative, that is, each inequality is certified.

Finally, Algorithm 1 and the test above can be implemented and solved by using packages like YALMIP, [16], and SeDuMi, [27].

\section{CONCLUSIONS}

We have shown a set of local conditions for studying the stability of a class of hybrid systems. Based on such conditions we proposed a sum of squares algorithm for characterizing the stability of such class hybrid systems. It could be of interest, as a future work, to consider an approach with piecewise functions, where several low-order polynomials are combined, instead of an approach based on a single high degree polynomial.

\section{APPENDIX}

\section{A. Proof of Theorem 2.}

We claim that, under Conditions (1)-(4) of Theorem 2, there exists a $\nu \in \mathbb{R}_{>0}$ and a $T \in \mathbb{R}_{>0}$ such that, for each solution $x$ to $\mathcal{H}$ with $|x(0,0)|=(1+\nu) c$,

- either $\operatorname{dom} x$ is compact and $\forall(t, j) \in \operatorname{dom} x, t+j \leq T$,

- or $\exists(t, j) \in \operatorname{dom} x$ such that $t+j \leq T$ and $|x(t, j)| \leq c$, and, in both cases, $\forall(s, i) \in \operatorname{dom} x$ such that $s+i \leq t+j$, $|x(s, i)| \leq \rho c$. Then, from [12, Proposition 4.3] $\mathcal{H}$ is globally pre-asymptotically stable ${ }^{1}$. Finally, from [5, Theorem 7.9] and [30, Theorem 2], (9) follows.

The proof of the claim can be developed as follows. For a sufficiently small $\nu \in \mathbb{R}_{>0}$ we have that

$$
\max _{|x|=(1+\nu) c} V(x)=\ell_{3}<\ell_{2} .
$$

The existence of such $\nu$ is guaranteed by $\ell_{1}<\ell_{2}$, by Condition (3), and by continuity of $V$.

${ }^{1}\left[12\right.$, Proposition 4.3] requires $|x(t, j)| \leq \frac{1}{2}(1+\nu) c$ instead of $|x(t, j)| \leq c$ in the or case. But the proof technique used in [12, Proposition 4.3] still works if we replace $\frac{1}{2}$ with any constant strictly smaller than 1 . Thus, the conclusion of [12, Proposition 4.3] is achieved also by using $|x(t, j)| \leq c$. 
From Conditions (1) and (2) and the fact that the set $\mathcal{S}=$ $\{x|c \leq| x \mid \leq \rho c\}$ is a compact set, there exist $\sigma_{1}, \sigma_{2} \in \mathbb{R}_{>0}$ such that (i) $\langle\nabla V(x), f\rangle \leq-\sigma_{1}$ for all $x \in C$ and each $f \in F(x)$, and (ii) $V(g)-V(x) \leq-\sigma_{2}$ for each $x \in D$ and each $g \in G(x)$. Then, for each solution $x$ to $\mathcal{H}$ with $c \leq|x(0,0)| \leq \rho c$,

$$
V(x(t, j)) \leq V(x(0,0))-\sigma(t+j)
$$

for all $(t, j) \in \operatorname{dom} x$, provided that $t+j \leq \inf \{s+i \mid(s, i) \in$ $\operatorname{dom} x$ and $x(s, i) \notin \mathcal{S}\}$ if $x$ leaves $\mathcal{S}$, and $\sigma=\min \left\{\sigma_{1}, \sigma_{2}\right\}$.

Moreover, consider $T \in \mathbb{R}_{>0}$ such that

$$
T>\frac{1}{\sigma}\left(\max _{|x|=(1+\nu) c} V(x)-\min _{x \in \mathcal{S}} V(x)\right) .
$$

Suppose now that the claim fails, that is, there exist a solution $x$ to $\mathcal{H}$ with $|x(0,0)|=(1+\nu) c$ and a time $(t, j) \in$ $\operatorname{dom} x$ such that $t+j \geq T$ and $|x(s, i)|>c$ for all $s+i \leq$ $t+j$, or for some $(s, i) \in \operatorname{dom} x,|x(s, i)|>\rho c$. Then, by condition (4) and $\ell_{3}<\ell_{2}$, for each $(s, i) \in \operatorname{dom} x, x(s, i)$ cannot jump to a point $g \in G(x(s, i))$ for which $|g|>\rho c$. Thus, (1), (2) apply and, with (3) and (4), they guarantee that the solution $x$ cannot escape $\mathcal{S}$ by reaching a point whose norm is greater than $\rho c$. Moreover, $V$ decreases along the solution $x$, bounded by (16).

Consider now (16). We have $V(x(s, i)) \leq \ell_{3}-\sigma(s+i)$ and suppose that for each $(s+i) \leq t+j,|x(s, i)|>c$. Then, for $s+i=T$,

$$
V(x(s, i)) \leq \ell_{3}-\sigma T<\min _{x \in \mathcal{S}} V(x) .
$$

Indeed, $\max _{|x|=(1+\nu) c} V(x)-\min _{|x| \leq c} V(x)=$ $\ell_{3}-\min _{|x| \leq c} V(x)$. Thus, $T$ is greater than $\frac{1}{\sigma}\left(\ell_{3}-\min _{x \in \mathcal{S}} V(x)\right)$, from which $\ell_{3}-\sigma T<\min _{x \in \mathcal{S}} V(x)$. Thus, $x(s, i) \notin \mathcal{S}$, therefore $|x(s, i)|<c$, which contradicts the hypothesis.

\section{B. Proof of Theorem 3.}

The if direction is a consequence of Theorem 2. The only if part can be proved as follows. By the converse result in [5, Theorem 3.14], for a a hybrid system $\mathcal{H}$ of Equations (1),(4)(8), if the equilibrium $x_{e}=0$ is globally pre-asymptotically stable, then there exists a smooth function $V: \mathbb{R}^{n} \rightarrow \mathbb{R}$ and $\alpha_{1}, \alpha_{2} \in \mathcal{K}_{\infty}$ such that

$$
\begin{array}{cl}
\alpha_{1}(|x|) \leq V(x) \leq \alpha_{2}(|x|) & \forall x \in \mathbb{R}^{n} \\
\langle\nabla V(x), f\rangle \leq-V(x) & \forall x \in C, \forall f \in F(x) \\
V(g) \leq e^{-1} V(x) & \forall x \in D, \forall g \in G(x) .
\end{array}
$$

Conditions (1), (2) of Theorem 2 are immediate.

(3) Choose a constant $\ell_{1}>0$ and define $c=\alpha_{2}^{-1}\left(\ell_{1}\right)$. Choose a constant $\ell_{2}>\ell_{1}$ so that $\rho=\alpha_{1}^{-1}\left(\ell_{2}\right) / c$ is strictly greater then 1. It follows that $\max _{|x|=c} V(x) \leq \alpha_{2}(c)=$ $\ell_{1}$ and $\min _{|x|=\rho c} V(x) \geq \alpha_{1}(\rho c)=\ell_{2}$. Condition (3) of Theorem 2 holds.

(4) Suppose now that there exists a $x \in D \cap\{x|c \leq| x \mid \leq$ $\rho c\} \cap\left\{x \mid V(x) \leq \ell_{2}\right\}$ and a $g \in G(x)$ such that $|g|>\rho c$. Then, $\ell_{2}=\alpha_{1}(\rho c)<\alpha_{1}(|g|) \leq V(g)$. Therefore $V(g)>$ $V(x)$, which contradicts (19). It follows that Condition (4) of Theorem 2 holds.

\section{Proof of Proposition 1.}

$V(x)$ is a polynomial function, so it is smooth.

(1) For each $i \in I_{C}$ and each $k=1, \ldots, r_{F}$, (10) can be written as $\nabla V(x) F_{i k} x<-\Delta_{2}^{(i)}(x)-s_{1}^{(i k)}(x) \Delta_{1}(c, \rho c, x)$. Therefore $\nabla V(x) F_{i k} x<0$ in $\left\{x|c \leq| x \mid \leq \rho c, x \in R^{(i)}\right\}$, for each $i \in I_{C}$ and $k=1, \ldots, r_{F}$. Suppose now that $x$ belongs to the intersection of some sets $R^{(i)}$, for $i \in I \subseteq I_{C}$. Then, for each $f \in F(x)$ we can write $\langle\nabla V(x), f\rangle$ as

$$
\left\langle\nabla V(x), \sum_{i \in I, k=1, \ldots, r_{F}} \lambda_{i k} F_{i k} x\right\rangle=\sum_{i \in I, k=1, \ldots, r_{F}} \lambda_{i k}\left\langle\nabla V(x), F_{i k} x\right\rangle
$$

for some $\sum_{i \in I, k=1, \ldots, r_{F}} \lambda_{i k}=1$, and $\lambda_{i k} \geq 0$ for each $i \in I$ and $k=1, \ldots, r_{F}$. It follows that $\langle\nabla V(x), f\rangle<0$ in $\{x|c \leq| x \mid \leq \rho c\} \cap C$, i.e. Condition (1) of Theorem 2 holds.

(2) (11) implies Condition (2). To see this, an argument similar to the one above on (10) can be used. No convex combination of vectors $G_{i k} x$ is needed in this case, in fact $g \in G(x)$ if and only if $g \in G_{i k} x$ for some $i \in I \subseteq I_{D}$ and $k=1, \ldots, r_{G}$.

(3) The first inequality in (12) can be written as $V(x) \leq$ $\ell_{1}-s_{3}(x) \Delta_{1}(c, c+\varepsilon, x)$, which implies $V(x) \leq \ell_{1}$ for $|x| \in[c, c+\varepsilon]$. It follows that $\max _{|x|=c} V(x) \leq \ell_{1}$. A similar argument can be used to show that the second inequality in (12) guarantees $\min _{|x|=\rho c} V(x) \geq \ell_{2}$. Therefore, Condition (3) of Theorem 2 is satisfied.

(4) For each $i \in I_{D}$ and each $k=1, \ldots, r_{G}$, Inequality (13) implies $V(x)-\ell_{2}-s_{5}^{(i k)}(x)\left(x^{\prime} G_{i k}^{T} G_{i k} x-\rho^{2} c^{2}\right) \geq 0$ for $x$ in $\left\{x\left|x \in R^{(i)}, c \leq\right| x \mid \leq \rho c\right\}$. We can write such inequality as $s_{5}^{(i k)}(x)\left(\rho^{2} c^{2}-x^{\prime} G_{i k}^{T} G_{i k} x\right) \geq \ell_{2}-V(x)$, and from $s_{5}^{(i k)}(x) \geq 0$, it follows that $\rho^{2} c^{2}-x^{\prime} G_{i k}^{T} G_{i k} x \geq 0$ for $x$ in $\left\{x\left|x \in R^{(i)}, c \leq\right| x \mid \leq \rho c, V(x) \leq \ell_{2}\right\}$. Note that $\rho^{2} c^{2}-x^{\prime} G_{i k}^{T} G_{i k} x \geq 0$ is equivalent to $\left|G_{i k} x\right| \leq \rho c$, and such relation hold for each $i \in I_{D}$ and each $k=1, \ldots, r_{G}$, whenever $x$ belongs to $\left\{x\left|x \in R^{(i)}, c \leq\right| x \mid \leq \rho c, V(x) \leq\right.$ $\left.\ell_{2}\right\}$. It follows that $|g| \leq \rho c$ for each $x$ in $\{x \mid x \in D, c \leq$ $\left.|x| \leq \rho c, V(x) \leq \ell_{2}\right\}$ and each $g \in G(x)$, i.e. Condition (4) of Theorem 2 is satisfied.

\section{REFERENCES}

[1] M. Badamchizadeh, S. Khanmohammadi, G. Alizadeh, A. Aghagolzadeh, and G. Karimian. Using sum of squares decomposition for stability of hybrid systems. IEICE Transactions, 90-A(11):2478-2487, 2007

[2] F. Blanchini and C. Savorgnan. Stabilizability of switched linear systems does not imply the existence of convex Lyapunov functions. Automatica, 44(4):1166 - 1170, 2008.

[3] M. Branicky. Multiple Lyapunov functions and other analysis tools for switched and hybrid systems. IEEE Trans. Aut. Cont., 43(4):475-482, April 1998.

[4] C. Cai, A.R. Teel, and R. Goebel. Smooth Lyapunov functions for hybrid systems-Part I: Existence is equivalent to robustness. IEEE Transactions on Automatic Control, 52(7):1264-1277, 2007.

[5] C. Cai, A.R. Teel, and R. Goebel. Smooth Lyapunov functions for hybrid systems part II:(pre) asymptotically stable compact sets. IEEE Transactions on Automatic Control, 53(3):734-748, 2008.

[6] P. Collins. A trajectory-space approach to hybrid systems. In International Symposium on the Mathematical Theory of Networks and Systems, Leuven, Belgium, July 2004.

[7] R.A. Decarlo, M.S. Branicky, S. Pettersson, and B. Lennartson. Perspectives and results on the stability and stabilizability of hybrid systems. Proceedings of the IEEE, 88(7):1069 -1082, jul 2000. 
[8] Schaft A. J. van der and J. M. Schumacher. Introduction to Hybrid Dynamical Systems. Springer-Verlag, London, UK, 1999.

[9] R. Goebel, J. P. Hespanha, A. R. Teel, C. Cai, and R. Sanfelice. Hybrid systems: Generalized solutions and robust stability. In Proc. of the 6th IFAC Symp. on Nonlinear Contr. Systems, Sep. 2004.

[10] R. Goebel, R. Sanfelice, and A. Teel. Hybrid dynamical systems. Control Systems Magazine, IEEE, 29(2):28-93, April 2009.

[11] R. Goebel and A.R. Teel. Solutions to hybrid inclusions via set and graphical convergence with stability theory applications. Automatica, 42(4):573 - 587, 2006.

[12] R. Goebel and A.R. Teel. Preasymptotic stability and homogeneous approximations of hybrid dynamical systems. SIAM Review, 52(1):87$109,2010$.

[13] T.A. Henzinger. The theory of hybrid automata. Logic in Computer Science, Symposium on, 0:278, 1996.

[14] M. Johansson and A. Rantzer. Computation of piecewise quadratic Lyapunov functions for hybrid systems. IEEE Transactions on Automatic Control, 43(4):555-559, Apr 1998.

[15] D. Liberzon. Switching in Systems and Control. Birkhäuser, 2003.

[16] J. Lofberg. YALMIP : A toolbox for modeling and optimization in MATLAB. In Proceedings of the International Symposium on Computer Aided Control System Design, Taipei, Taiwan, 2004.

[17] J. Lofberg. Pre- and post-processing sum-of-squares programs in practice. IEEE Transactions on Automatic Control, 54(5):1007-1011, May 2009.

[18] J. Lygeros, K. H. Johansson, S. N. Simic, J. Zhang, and S. Sastry. Dynamical properties of hybrid automata. IEEE Transactions on Automatic Control, 48:2-17, 2003.

[19] A. Papachristodoulou and S. Prajna. On the construction of Lyapunov functions using the sum of squares decomposition. In Proceedings of the 41st IEEE Conference on Decision and Control, 2002, volume 3, pages 3482-3487 vol.3, Dec. 2002.

[20] A. Papachristodoulou and S. Prajna. Robust stability analysis of nonlinear hybrid systems. IEEE Transactions on Automatic Control, 54(5):1035-1041, May 2009.

[21] P.A. Parrilo. Structured Semidefinite Programs and Semialgebraic Geometry Methods in Robustness and Optimization. $\mathrm{PhD}$ thesis, California Institute of Technology, 2000.

[22] S. Prajna and A. Papachristodoulou. Analysis of switched and hybrid systems - beyond piecewise quadratic methods. In Proceedings of the 2003 American Control Conference, volume 4, pages 2779-2784 vol.4, June 2003.

[23] S. Prajna, A. Papachristodoulou, P. Seiler, and P.A Parrilo. SOSTOOLS and its control applications. In D. Henrion and A. Garulli, editors, Positive Polynomials in Control, volume 312, pages 273-292. Springer Verlag, 2005.

[24] R.J.-B. Wets. M. Rockafellar, R.T. Wets. Variational Analysis. Springer, 2004.

[25] R.G. Sanfelice, R. Goebel, and A.R. Teel. Invariance principles for hybrid systems with connections to detectability and asymptotic stability. IEEE Transactions on Automatic Control, 52(12):2282-2297, 2007.

[26] R.G. Sanfelice, R. Goebel, and A.R. Teel. Generalized solutions to hybrid dynamical systems. ESAIM: COCV, 14(4):699-724, Oct 2008.

[27] J. F. Sturm. Using SeDuMi 1.02, a MATLAB toolbox for optimization over symmetric cones. Optimization Methods and Software, 1112:625-653, 1999

[28] Z. Lin T. Hu, L. Ma. Stabilization of switched systems via composite quadratic functions. IEEE Trans. Aut. Cont., 53(11):2571-2585, December 2008.

[29] W. Tan. Nonlinear Control Analysis and Synthesis using Sum-ofSquares Programming. PhD thesis, University of California, Berkeley, 2006.

[30] S.E. Tuna and A.R. Teel. Homogeneous hybrid systems and a converse Lyapunov theorem. In 45th IEEE Conference on Decision and Control, 2006, pages 6235-6240, Dec. 2006. 